\title{
The mystical formula and the mystery of Khronos
}

\author{
Orfeu Bertolami \\ Instituto Superior Técnico, Departamento de Física, \\ Av. Rovisco Pais 1, 1049-001, Lisboa, Portugal \\ orfeu@cosmos.ist.utl.pt
}

\begin{abstract}
In 1908, Minkowski put forward the idea that invariance under what we call today the Lorentz group, $G L(1,3, \mathbf{R})$, would be more meaningful in a fourdimensional space-time continuum. This suggestion implies that space and time are intertwined entities so that, kinematic and dynamical quantities can be expressed as vectors, or more generally by tensors, in the four-dimensional space-time. Minkowski also showed how causality should be structured in the four-dimensional vector space. The mathematical formulation proposed by Minkowski made its generalization to curved spaces quite natural, leaving the doors to the General Theory of Relativity and many other developments ajar.

Nevertheless, it is remarkable that this deceptively simple formulation eluded many researchers of space and time, and goes against our every day experience and perception, according to which space and time are distinct entities. In this contribution, we discuss these contradictory views, analyze how they are seen in contemporary physics and comment on the challenges that space-time explorers face.
\end{abstract}

\section{The mystical formula}

On the 21st of September 1908, at his address to the 80th Assembly of German Natural Scientists and Physicians in Cologne, Hermann Minkowski (1864 1909) presented his World Postulate, Weltpostulat, according to which the invariance under what we call today the Lorentz group $G L(1,3, \mathbf{R})$, would be more meaningful in a 4-dimensional space-time continuum [1. This follows the very spirit of special relativity, in that the independence of the laws of physics on the velocity of inertial frames requires that space and time are indissoluble concepts and are related to each other by the maximum attainable particle velocity. In his own words: 
"The views of space and time which I wish to lay down for you have sprung from the soil of experimental physics, and therein lies their strength. They are radical. Henceforth space by itself, and time by itself, are doomed to fade away into mere shadows, and only a kind of union of the two will preserve an independent reality."

To further stress the somewhat unusual nature of his proposal, Minkowski simply states that "no one can ever refer to a Space without its Time or to a Time without its Space" and actually, much more emphatically, "the essence of the World Postulate, which is pregnant with mathematical implications, could be dressed in the mystical formula:"

$$
3 \times 10^{5} \mathrm{~km}=\sqrt{-1} \mathrm{sec} .,
$$

where the imaginary unit arises as Minkowski chooses to view space-time as strictly Euclidean, in its signature and in its lack of curvature. Thus, an event in the space-time continuum should be referred to as a world-point, "Welt-punkt", while its evolution in space-time continuum through a worldline, "Welt-linie". Thus, according to Minkowski, "all the world presents itself quite explicitly through world-lines" to the point that in his opinion, "physical laws would find their most comprehensive formulation through the reciprocal relationships of world-lines".

It is clear that the suggestion of a space-time continuum represents a further unification of concepts in physics (for a discussion on the inconsistency of a 3-dimensional world see Ref. 2 ). Indeed, special relativity allowed for a unified description of the laws of physics as well as for an unique formulation of mass, energy and momenta, thanks to the invariance of the maximum attainable particle velocity, $c_{S T}$, the Relativity Principle. It is important to remember that from the Relativity Principle, in any physical setting, distances can be measured by clocks and mirrors. Furthermore, an immediate implication of this order of ideas is that if space is isotropic, then any attempt to measure the time difference in the time of travel of light of equal distance paths would yield, irrespective of the direction, a null result. The most recent experimental attempts to measure deviations from this null outcome have shown that it holds up to a few parts in $10^{-9}$ [3. Indirect experiments involving, for instance, ultra high-energy cosmic rays yield even more impressive limits, actually $1.7 \times 10^{-25}$ (see e.g. Ref. [4] and references therein).

At this point, two comments are in order. The first one refers to the fact that the identification of the maximum attainable particle velocity with the speed of light, $c$, is only possible because, up to the current experimental precision, the photon mass vanishes and electromagnetism is an exact abelian gauge theory. If this were not the case, these two velocities could not be the same. Of course, historically, these two velocities were not initially distinguished and current bounds on the photon mass are compatible with this identification (see for instance [5]). Naturally, the same can be said about the identification of the speed of light and the velocity of propagation of gravitational waves in 
vacuum [6]. Thus, given the present bounds on the the photon (and also the graviton) mass we shall simply identify $c_{S T}$ with $c$.

The second comment refers to the fact that the Standard Model (SM) vacuum being non-trivial, might not respect Lorentz invariance and hence correspond to a sort of preferred frame [7. Alternatively, one can consider that only particle Lorentz invariance is physically meaningful, a perspective which allows for an extension of the SM compatible with the spontaneous breaking of Lorentz invariance, without implying the existence of a preferred frame [8. Of course, this possibility would lead to distinct experimental signatures, which so far have not been observed (see e.g. Refs. [9] for comprehensive discussions).

So according to Minkowski, the motion of particles in the space-time continuum correspond to lines, world lines, from a given point in space-time where the original event took place. Past and future and hence causality are referred to this original event. In terms of the metric

$$
d s^{2}=-c^{2} d t^{2}+d x^{2}+d y^{2}+d z^{2},
$$

space-time admits light-like world lines, for which $d s^{2}=0$, time-like interval, for which $d s^{2}<0$, and a space-like interval, for which $d s^{2}>0$. Thus, in spacetime diagrams, where time is depicted in the vertical axis and space in the horizontal one, light travels in the cone, the light-cone or null curves described, for a given time interval $\Delta t$, by $c \Delta t= \pm \sqrt{\Delta x^{2}+\Delta y^{2}+\Delta z^{2}}$. Events within this cone are time-like and given that observers move with a relative velocity $v<c$, word-lines within this cone connect events in the past or in the future of each other, whether they precede or succeed each other. An event, say $A$, outside the light-cone cannot influence or be influenced by any other event separated by $A$ by a space-like interval.

Clearly, these relationships have an absolute and global nature given the independence of the velocity of light on the velocity of the frame of reference; however, special relativity makes the concept of an absolute simultaneity impossible and thus the idea of an universal present. Of course, as already mentioned, this together with the fact that time flows at different rates for different observers and that likewise, the perception of space is also tied up with the relative motion of different observers, drives one away from Newton's (1643 - 1727) conception of absolute time, defined in the first book of his Principia Mathematica in 1687: "Absolute, true, and mathematical time, in and of itself and of its own nature, without reference to anything external, flows uniformly and by another name is called duration".

The insightful formulation of Minkowski allowed for a straightforward generalization and that was the path followed by Einstein (1879 - 1955) from 1907 onward after realizing that Newtonian gravity did not fit within the framework of special relativity. Later on, in collaboration with his friend and Zurich's Technical University colleague, Marcel Grossmann (1878 - 1936), Einstein wrote a seminal paper, albeit not quite consistent, in 1913, where it was clearly spelled out that Riemannian geometry was actually the most general 
and natural setting for physics (see, for instance, Ref. 10 for a detailed account). Of course, these developments relied strongly on the XIX century work of Lobatchevski(1793 - 1856), Bolyai (1802 - 1866), Riemann (1826 - 1846) and Gauss (1777 - 1855), who have shown that flat spaces are a particular case of a much wider class of spaces with non-vanishing curvature. This was indeed a quite new idea, even though, space and time of day to day affairs, were still regarded as a priori concepts that preceded all experience and were independent of any physical phenomena in the Newtonian (and Kantian) sense. But, of course, general relativity revolutionized this view showing that space and time are actually associated with a given energy-matter distribution, so that the Newtonian perspective was, at best, just an approximation to the inner nature of space and time.

Actually, the notion of world-lines in general relativity is basically similar to the one in special relativity, with the difference that in the former, spacetime can be curved. The dynamics of the metric is determined by the Einstein field equations and depends on the energy-mass distribution in space-time. As before, the metric defines light-like (null), space-like and time-like curves. Also, in general relativity, world lines are time-like curves in space-time, where timelike curves fall within the light-cone. However, a light-cone is not necessarily inclined at 45 degrees to the time axis, if one adopts the unit system where $c=1$. However, this is an artifact of the chosen coordinate system, and reflects the coordinate freedom, the very essential diffeomorphism invariance of general relativity. Any time-like curve admits a co-moving observer whose "time axis" corresponds to that curve, and, since no observer is privileged, we can always find a local coordinate system in which light-cones are inclined at 45 degrees to the time axis. Furthermore, the world-lines of free-falling particles or objects, such as the ones of planets around the Sun or of an astronaut in space, are minimal length curves, the so-called geodesics.

However, one should keep in mind that general relativity contains geometries which defy the very core of physical reasoning. Indeed, the existence of closed time-like curves contradicts the essential features of causality and chronology. Moreover, singularities, unavoidable in general relativity, once closed time-like curves are absent and geometry is set by well-behaved matter-energy configurations, imply that geodesics cannot exist in the whole space-time.

The referred condition on matter-energy is fairly specific as in the HawkingPenrose singularity theorems and is tied up with the physical nature of a manifold [11. A Lorentzian manifold $(M, g)$ is said to be physically well-behaved if it satisfies the strong energy condition:

$$
R_{\mu \nu} V^{\mu} V^{\nu} \geq 0,
$$

for any time-like vector field, $V^{\mu}$. From Einstein's equations this statement is equivalent, for more than two $d$-spatial dimensions, to the condition on the energy-momentum tensor and its trace, $T$, 


$$
T_{\mu \nu} V^{\mu} V^{\nu} \geq \frac{T}{d-1} V_{\mu} V^{\mu} .
$$

This condition is fulfilled by spaces dominated by the vacuum with a positive cosmological constant $(\Lambda \geq 0)$, and by a perfect fluid if $\rho+3 p \geq 0$, where $\rho$ and $p$ correspond to the energy density and isotropic pressure, respectively.

Of course, the fundamental assumption here is the connection between physical spaces with mathematical spaces that satisfy the Einstein field equations. A generic mathematical space is fundamentally described by a $d$-dimensional differentiable manifold $M$ endowed with a symmetric, nondegenerate second-rank tensor, the metric, $g$. A manifold under these specifications is said to a pseudo-Riemannian manifold, $(M, g)$, as it has a Lorentzian signature $(-,+, \ldots,+)$ - it is Riemannian if it has signature $(+, \ldots,+)$. A differentiable manifold admits a Lorentzian signature if it is noncompact or has a vanishing Euler characteristic. The Italian mathematician Tullio Levi-Civita (1873 - 1941), showed a well known theorem according to which a pseudoRiemannian manifold has a unique symmetric affine connection compatible with the metric, being hence equipped with geodesics.

The spaces relevant to physics correspond to solutions of the Einstein equations with a cosmological constant 1 , $\Lambda$ :

$$
R_{\mu \nu}-\frac{1}{2} g_{\mu \nu} R+\Lambda g_{\mu \nu}=8 \pi G T_{\mu \nu},
$$

where $R_{\mu \nu}$ is the Ricci curvature of $M, R$ its trace, $G$ is Newton's constant and $T_{\mu \nu}$ is the energy-momentum tensor of matter in $(M, g)$.

Thus, through Minkowski unification, it was possible to intimately relate physics back to geometry, a connection quite dear to Galileo (1564 - 1642) and Newton, but somewhat lost in the XIX century physics. In a previous text by this author [21, this methodology was referred to as "Cézanne's principle", given the suggestive connection it has with the writings of the French painter who first reflected on the then new cubist revolution. According to Cézanne (1839-1906), the essence of the new movement was the description of nature through purely geometrical forms. One could further mention that the relativity revolution is seen by many thinkers to be somewhat similar to the one that took place in modern art through movements like futurism, cubism, and other "isms" which have shown to be possible to depict in a single plane various points of view, as well as the mutation of reality through superimposing images. In this way, time was introduced into the arts that were traditionally associated with space, such as sculpture, architecture and painting, in opposition to the ones associated with time, music and literature.

The fundamental insight of Minkowski allowed for the generalization of the special theory of relativity and its application for understanding the inner secrets of the microscopical world through the development of quantum field theory. As far as experimental evidence allows us to unravel, quantum field

\footnotetext{
${ }^{1}$ The "natural" system of units is used: $c=\hbar=k_{B}=1$.
} 
theory methods are consistent down to about $10^{-18} \mathrm{~m}$. On the largest known scales, general relativity, where the most general space-time continuum is actually curved, cosmological evidence seems to fit the so-called cosmological standard model up to the horizon size, i.e. distances up to $10^{26} \mathrm{~m}$ (see e.g. 13, 14). This is an impressive vindication of Minkowski's World Postulate.

\section{The mystery of Kronos}

If at the physical and conceptual level, one could assume that most of the merit of the Minkowski unification is due to the radically different view implied by special relativity a few years earlier thanks to the work of Einstein, one should realize that from the historical and philosophical standpoint, the proposal of Minkowski is a remarkable culmination of more than two thousand years of research about the nature of space and time. In what follows we shall discuss some of the most conspicuous philosophical ideas about the space and time problematic. Our main sources for the ensuing discussion are Refs. [1, 16, 17, 18, 19, 20, 21, 22.

The very first manifestations of articulate rational thinking about the origin of the world, myths of creation, often regarded space and time as inseparable, as the original creation act gave birth to both space and time - actually, likewise the modern theory of the Big-Bang. In ancient Hellenic period, space and time were seen as two essential features of reality, but in many instances, regarded as distinct entities.

Indeed, at first, space and time seem to be quite different. Space can be freely experienced as one can move in any direction without restriction. Time however, has a well defined direction. Past and future are clearly distinct as our action can affect only the latter. We have memory, but not precognition. Matter, organic or otherwise, tends to decay rather than to organize itself spontaneously. There seems to exist at least 3 distinct spatial dimension $2^{2}$ while there is only a single time dimension. Actually, the fact that

\footnotetext{
${ }^{2}$ The Finnish physicist Gunnar Nordström (1881 - 1923) was the first to speculate in 1909, that space-time could very well have more than four dimensions. A concrete realization of this idea was put forward by Theodor Kaluza (1885 - 1954) in 1919 and Oskar Klein (1894 - 1977) in 1925, who showed that an unified theory of gravity and electromagnetism could be achieved through a 5-dimensional (4spatial and a single time dimension) version of General Relativity. These extra dimensions in order to have passed undetected can be either compact and very small or very large if the known fundamental interactions, excluding gravity, can test only 3-spatial dimensions. In any case, the extension of the number of spatial dimensions has been widely considered in attempts to unify all known four interactions of nature. For instance, the requirement that supersymmetry is preserved in 4 dimensions, from the original 10-dimensional superstring theory, implies that 6 dimensions of the world are compact 23 . Connecting all string theories through $S$ and $T$ dualities suggests the existence of an encompassing theory, M-theory, and that space-time is 11-dimensional [24].
} 
in many physical theories the time dimension is just a parameter turns it into an "invisible dimension" 26. Notice that if the number of time dimensions is greater than one, one expects all type of complications as, on quite general grounds, the Partial Differential Equations that describe the physical phenomena are ultra-hyperbolic, which leads to unpredictability, or in weird "backward causality" (see e.g. Ref. [12] for a discussion).

Let us resume the philosophical discussion. Space has always been regarded as the arena of all manifestations of nature. Everything lies in space and the intrinsic and fundamental relationships between the most basic elements of everything could be decomposed into points, straight lines and geometrical figures in two or three dimensions and whose properties were monumentally described by Euclid's (ca. 330 - 275 B.C.) geometry. These relationships would in turn reveal the intrinsic properties of space itself. Of course, the fundamental role of space in the Hellenic philosophical thinking was more than evident on the speculative thinking of the pre-Socratic Zeno (495 - 435 B.C.) and Pythagoras (ca. 569 - 500 B.C.) and many others after them. For instance, for Plato (428 - 349 B.C.), "God ever geometrizes". For Aristotle (384 - 322 B.C.), the "geometrical" method and proof was the intellectual reasoning model that should be used in natural sciences, ethics, metaphysics and so on.

There were however, instances where philosophical thinking hinted at a hidden connection between space and time. For instance, for Zeno, the Dichotomy, the Achilles - tortoise, and the Arrow paradoxes stressed the fundamental difficulty in reconciling motion, that is dislocation in space, actually along a straight line, with the concepts of continuity and divisibility. In his Stadium paradox, Zeno considers three rows of bodies lying on a line and how the opposite relative motion of two of the rows "proof that half the time may be equal to double the time" 28. Of course, these puzzles reflect the immaturity of the mathematical thinking at Zeno's time concerning the infinitesimal. But, it is suggestive that Zeno was already seeing that paradoxes in space and time were related in the real physical world through motion. A contemporary physicist could not fail to see that the divisibility process considered by Zeno could not go on indefinitely, as the fundamental limitation of quantum effects on the fabric of space-time would arise at Planck length, $10^{-35}$ meters (or equivalently Planck time level, $10^{-44}$ seconds), the length where the Schwarzschild radius of a particle equals its Compton wavelength 3 .

For Pythagoras, who was the first to understand that above the application of mathematical tomb rules stood the proof of the fundamentals behind the rules, one could argue that the association of mathematics with music implied

\footnotetext{
${ }^{3}$ Actually, in some quantum gravity approaches, as for instance in loop quantum gravity, space-time is suggested to have, at its minutest scale, presumably the Planck scale, $L_{P} \simeq 10^{-35} \mathrm{~m}$, a discrete structure [25]. In superstring/M-theory, the space-time continuum is an emergent property that arises from the ground state excitations of closed strings, one of the fundamental objects of the theory.
} 
an inner connection between geometry and tempo, the very essence of music, time.

Actually in the Hellenic mythology, more specifically in the Orphic cosmogonies, time had a particularly interesting standing. Khronos, the primeval god of time, emerged as a self-formed divinity at the beginning of creation [29]:

"Originally there was Hydros (Water) and Mud, from which Ge (Earth) formed solidified ... The third principle after the Hydros and Ge was engendered by these, and was a Drakon (Serpent) with extra heads of a bull and a lion and a god's countenance in the middle; it had wings upon its shoulders, and its name was Khronos (Unaging Time) and also Herakles. United with it was Ananke (Inevitability, Compulsion), being of the same nature, her arms extended throughout the universe and touching its extremities ..."

Thus, Khronos and Ananke encircled the cosmos from the time of creation, and their passage drives the circling of heaven and the eternal flow of time.

¿From myth to rational thinking, time was insightfully dissected by Heraclitus (ca. 535 - 475 B.C.) and by Aristotle (384 - 322 B.C.). Heraclitus understood the world as a unit resulting from diversity in eternal transformation. Time is what allows events to occur as a result of a web of antinomies. The formulas:"You cannot step twice in the same river; for fresh waters are ever flowing in upon you" and "The sun is new every day", capture the powerful idea that "all things are flowing".

For Aristotle, "time is a measure of motion according to the preceding and the succeeding", time is associated to the evolution, change in quantity and/or quality, of all occurrences in nature. For the most influential disciple of Plato, time is intimately related to motion, and with the counting process that is, with numbers. Aristotle's view leads to an operational connection between time and any material system that can be used as a standard for measuring the passage of time: clepsydras, sand clocks, sun clocks, pendulums and clocks. Since ancient times, the motion of earth around the sun has been the measure of day to day activities. Thus, in essence, our closest connection to time is the very one put forward by Aristotle more than two thousand years ago. This is irrespective of any technological development, whether we use the regularity of the astronomical motions in the solar system (which, of course, cannot be exact due to the complexity of the physics behind these motions) or atomic clocks, or even binary pulsars, possibly the most precise clocks in the universe. In practical terms, one defines the second, the fundamental unit of time, as $1 / 86400$ of the duration of the average solar day, or 9, 192,631, 770 periods of transition the radiation corresponding to the transition between the two hyperfine levels of the ground state of the cesium-133 atom 4 .

\footnotetext{
${ }^{4}$ This definition concerns a cesium atom at rest at a temperature of $0 K$, such that the ground state is defined at zero magnetic field.
} 
The average solar motion is defined in terms the of the idealized uniform motion of the sun along the celestial equator. The difference between this idealized motion and the real motion is called "equation of time".

Coming back to the philosophic discussion, the vision of time by Saint Augustine (354 - 430) is remarkable in its modernity. He believed that the origin of time was the creation of the world. This was a fundamental precaution since God's eternity made its identification with time impossible. In his Confessions he expresses his view that the notion of present is the most fundamental feature of time:

"The present of things past is memory. The present of things at present is perception. The present of things in the future is expectation."

Another fundamental aspect of Saint Augustine's view of time is his rejection of the doctrine of cyclic history. In many ancient civilizations, Sumer, Babylon, Indian, Mayan, the regular patterns of tides, seasons and the cyclic motion of heavenly bodies entailed from the fact that time itself was circular. Day is followed by night, night by day, summer follows winter, winter by summer, old moon follows new, new one by old one, and so why not history itself ?

The cyclic temporal pattern was a noticeable feature of Greek cosmology. The Stoics believed that on every instance after the planets returned to their exact relative positions as at the beginning of time, the whole cosmos would become renewed. Nemesius, Bishop of Emesa says at the Vth century: "Socrates and Plato and each individual man will live again ... And this restoration of the universe takes place not once, but again and again, indeed to all eternity without end". The Maya civilization of Central America believed that history would repeat itself every 260 years, a period of fundamental importance in their calendar. The ancient Indians (Hindus, Budhists, Jains) extended the idea of a Great Year, a full cycle, into a hierarchy of Great Years. The destruction and re-creation of individuals and creatures occurred in a day of Brahma. A day of Brahma lasted 4 thousand million years. The elements themselves and all forms will undergo a dissolution into Pure Spirit, which then incarnates itself back into matter every lifetime of Brahma, that is, about $311 \times 10^{12}$ years. The lifetime of Brahma is the longest cycle and is repeated ad infinitum (see e.g. in [30] and references therein).

The notion of cyclic time was thus regarded more comfortable, as a time arrow would mean instability, and inevitably irreversible change. The myth of eternal return was a central idea of many ancient civilizations, exceptions being the Hebrews and the Zoroastrian Persians. Thus, it was through the Judaeo-Christian tradition and most definitely through Saint Augustine that linear and irreversible time got established in the Western culture. According to Saint Augustine, Christ's death and Crucification was an unique event, and from then on the cultural prominence of the Roman Christian Church took charge of "spreading the word" about the linear nature of time and history. But even before that it was considered heresy to claim otherwise and transgressors were being punished in an exemplary way. Despite that, defenders 
of the doctrine of circular time, sometimes referred to as the annulars, were not short of conviction. In the III century, Euforbo, a presumed member of the annulars sect, while burnt at the stake, is alleged to have screamed: "This happened already and will happen again. You do not light a pyre, you light a labyrinth of fire. If all the pyres that I have been were put together they would not fit on earth and would blind the angels. I have said that many times" 31.

In this context, it is particularly interesting to remark that Spinoza (1632 1677), who believed that everything could be attributed to a manifestation of God's inscrutable nature, and as such occurred by absolute logical necessity, defended the idea that time was unreal, and hence all emotions associated to an event in the future or in the past are contrary to reason. For this philosopher, whose ethics should follow as in Euclid's geometry from definitions, axioms and theorems, it should be understood that "in so far as the mind conceives a thing under the dictate of reason, it is affected equally, whether the idea be of a thing present, past or future".

The existence of physical time was also doubted by the 11th century, Persian philosopher Avicenna, who argued that time exists only in the mind due to memory and expectation. It is remarkable that similar views were expressed by Einstein himself. Indeed, in a letter to his life long friend, Michelle Besso, he writes (see e.g. [26, 27]:

"There is no irreversibility in the basic laws of physics. You have to accept the idea that subjective time with its emphasis on the now has no objective meaning".

Later, on the occasion of Besso's passing away, in a letter addressed to his widow and son, he says:

"Michelle has preceded me a little in leaving this strange world. This is not important. For us who are convinced physicists, the distinction between past, present and future is only an illusion, however persistent".

Also worth mentioning is the view of time of one of the most brilliant opponents of Newton, Gottfried Leibniz (1646 - 1716). He argued that time cannot be an entity existing independently of actual events. For Leibniz absolute space does not exist. Space is the relative configuration of bodies that exist simultaneously. Thus, time is the succession of instantaneous configurations, and not a flux independent of the bodies and their motion. It follows that as time concerns a chronology of events, in a universe where nothing happens, there is no time. This disagreement with the very basis of Newtonian mechanics lead Leibniz to suggest that mechanics should be built strictly in terms of observed elements. This view was shared by the science philosopher Ernest Mach (1838 - 1916) and Heinrich Rudolph Hertz (1857 - 1894), who actually developed a "relational" mechanics based on the ideas of Leibniz. It is well known that Einstein was particularly impressed by Leibniz's ideas on space and time and these inspired him when constructing the general theory of relativity, and also played an important role in his life long rejection of quantum mechanics. 
Causation, and thus time ordering, is according to Hume (1711 - 1776) the basis of human understanding. He warns that: "We ought not to receive as reasoning any of the observations we make concerning identity, and the relations of time and place; since in none of them the mind can go beyond what is immediately present to the senses ... Causation is different in that it takes us beyond impressions of our senses, and informs us of unperceived existences." It is arguable whether on purely philosophical terms Hume's doctrine stands on its own; however the decomposition of human perception down to the physiology of nervous tissues, down to its chemistry and then primarily the causal character of the physical laws, render Hume's proposition quite plausible.

In the Critic of Pure Reason, first published in 1781, Immanuel Kant (1724 - 1804) advances ideas about space, time and actually the main metaphysical problems of his time that turned out to be particularly influential. For Hume, the law of causality is not "analytic", that is, a proposition in which the predicate is part of the subject, such as for instance, an "equilateral triangle is a triangle". Kant agreed in that causation was a crucial starting point, however for him this law is synthetic and known a priori. A "synthetic" proposition is the one that is not analytic. All propositions that we know only through experience are synthetic. An "empirical" proposition is one which we cannot know except through the sense-perception, either our own or that of someone's testimony. So are the facts of history and geography, as well as the laws of science in so far as our knowledge of their truth depends on observational data. An a "priori" proposition, on the other hand, although susceptible of elucidation by experience, has, after inspection, a basis other than experience. All the propositions of pure mathematics are a priori. Kant then poses the question: How are synthetic judgments a priori possible ? His solution can be expressed in the following way: The outer world can only excite our senses, but it is our own mental apparatus that orders our sensations in space and time, providing in this way the means through which we understand experience. Space and time are thus subjective, they are part of our apparatus of perception; however, precisely because of their a priori nature, whatever we experience will exhibit the features that can be dealt with through geometry and the science of time. Space and time, Kant argues, are not concepts; they are means of intuition, forms of viewing or looking at the world.

Of some interest to physic:5 is also the part of the Critic of Pure Reason which deals with the fallacies, the "antinomies", mutually contradictory propositions which can be both proved to be true. They that arise from applying space and time or the categories (things in themselves) to what cannot be experienced. Kant discusses four of such antinomies, each consisting of thesis

\footnotetext{
${ }^{5}$ One should keep in mind that in 1755 Kant anticipated, in his General Natural History and Theory of the Heavens, how from Newton's mechanics one could explain the origin of the solar system. This nebular hypothesis was actually made mathematically plausible by Laplace's (1749 - 1827) many decades later.
} 
and antithesis. The first states: "The world has a beginning in time, and is also limited as regards to space". The antithesis says: "The world has no beginning, and no limits in space; it is infinite in regard to both time and space." The second antinomy proofs that every composite substance is both, and is not, made up of simple parts. The third antinomy states that there are two kinds of causality, one associated to the laws of nature, the other concerning that of freedom. The antithesis maintains that there is only the causality related to the laws of nature. Finally, the fourth antinomy shows that there is, and there is not, an absolute necessary Being. In a subsequent section, Kant destroys all purely intellectual proofs of the existence of God, even though he clarifies that he has other reasons for believing in God.

The antinomies have greatly influenced another important German philosopher, George Friedrich Hegel (1770 - 1831). Hegel believed in the unreality of the separateness, whether atoms or souls. The world is not a collection of self-subsistence units; nothing, Hegel held, is ultimately and completely real except the whole. Related to this is his disbelief in the reality of space and time as such, as these, if taken as completely real, involve separateness and multiplicity, which he regards as an illusion or as a mystic insight. Wholeness is the reality and this is rational as the rational is real. The engine of his metaphysical view of the world was dialectic: a thesis, antithesis and synthesis which sets consistency with the whole. His dialectic method applied to history in his Philosophy of History, could arguably give unit, and meaning to revolutions and movements of human affairs at the level of ideological currents of thought. Atributable to Karl Marx (1818 - 1883) is the theory that actually, the ultimate cause of human affairs moves dialectically, due to the clash of conflicting means of economical production. It is interesting that Marx regarded his insight about the development of human society as being analogous to the teleological evolution of species, the engine and clock of biological change, as first proposed by Darwin (1809 - 1882) and Alfred Russel Wallace (1823 - 1913) in 1858.

For Henri Bergson (1859 - 1941), intelligence and intellect can only form a clear idea of discontinuity and immobility, being therefore unable to understand life and to think about evolution. Intellect tends to represent becoming as a series of states. Geometry and logic, the typical products of intelligence, are strictly applicable to solid bodies, but to everything else reasoning must be checked by common sense. Actually, Bergson believes that the genesis of intelligence and the origin of material bodies are correlative and have been developed by reciprocal adaptation. For him, the growth of matter and intellect are simultaneous. Intellect is the power of seeing things as separate and matter is what is separated into distinct things. However, in reality, there are no separate solid things, only a continuous stream of becoming. Becoming being an ascendant movement that leads to life, or a descendant movement leading to matter.

For Bergson, space and time are profoundly dissimilar. The intellect is associated with space, while instinct and intuition are connected with time. 
Space, the characteristic of matter, arises from a dissection of the flux which is really illusory, although useful in practice. Time, on the contrary, is the essential feature of life or mind. "Wherever anything lives, there is, open somewhere a register in which time is being inscribed". But time here is not a "mathematical" time, an homogeneous assemblage of mutually external instants. Mathematical time, according to Bergson, is actually a form of space; the time which is the essence of life is what he refers to as duration. "Pure duration is the form which our conscious states assume when our ego lets itself live, when it refrains from separating its present state from its former states". Duration unites past and present into an organic whole, where there is mutual entanglement and succession with distinction.

We could not draw to an end this brief account on the philosophical thinking about the nature of space and time without a reference to the images that often arise in poetry and literature, where time in particular, is quite often insightfully evoked. Indeed, from the Rubáiyát of Omar Khayyám (1048 1131) to "La recherche du temps perdu" of Marcel Proust (1871 - 1922), from William Blake (1757 - 1827) to contemporary authors such as Imre Kertész and Paul Auster, time and memory are central themes in the literary context. One often finds quite profound glimpses on the nature of time. In "Du côte de chez Swann", Proust says:

"The past is hidden beyond the reach of intellect, in some material object (in the sensation that the object will give us). And as for that object, it depends on chance whether we come upon it before we ourselves die."

Actually, this fundamental impression which allows us to expand our imagination and build theories based on the discovery of these "time capsules", a rock containing a fossil, the light of a distant star, the cosmic microwave background radiation or an ancient picture.

Time, the cycles of life and the hope of prevalence as put forth by Shakespeare (1564 - 1616) in his LX sonnet [32]:

Like as the waves make towards the pebbled shore, So do our minutes hasten to their end;

Each changing place with that which goes before, In sequent toil all forwards do contend.

Nativity, once in the main of light,

Crawls to maturity, wherewith being crown'd,

Crooked eclipses 'gainst his glory flight,

And Time, that gave, doth now his gift confound.

Time doth transfix the flourish set on youth,

And delves the parallels in beauty's brow;

Feeds on the rarities of nature's truth,

And nothing stands but for his scythe to mow:

And yet to times in hope my verse shall stand, Praising thy worth, despite his cruel hand. 


\section{Arrows of time}

By the second half of XIX century, the development of the kinetic theory of matter by Maxwell (1831 - 1879), Clausius (1822 - 1888) and Boltzmann (1844 - 1906) revived once again the discussion on the dichotomy between the linear evolution of time and the eternal recurrence of motion.

The idea of a cyclic time and of an eternal return was recovered in philosophy by Herbert Spencer (1820 - 1903) and Friedrich Nietzsche (1844 - 1900) about the same time that Poincaré (1854 - 1912) showed his well known recurrence theorem. For sure, their "proofs" cannot be considered rigorous by the standards of physics and mathematics; however, interestingly, the "proof" of Nietzsche contains elements which can be regarded as relevant for any discussion of the issue, such as a finite number of states, finite energy, no creation of the universe and chance-like evolution. In his Dialectic of Nature, the philosopher and revolutionary politician, companion and co-author with Karl Marx of the Communist Manifesto, Friedrich Engels (1820 - 1895) wrote in 1879:

"... an eternal and successive repetition of worlds in an infinite time is the only logical conclusion of the coexistence of countless worlds in an infinite space ... It is in an eternal cycle that matter moves itself. "

Let us now turn to the physical discussion. Newton's equations have no intrinsic time direction, being invariant under time reversal; however, Poincaré showed in 1890, in the context of classical mechanics, a quite general recurrence theorem, according to which any isolated system, which includes the universe itself, would return to its initial state given a sufficiently long time interval.

Poincaré's theorem is proved to be valid in any space $X$ on which there exists a one parameter map $T_{i}$ from sets $[U]$ and a measure $\mu$ on $X$ such that: i) $\mu(X)=1$ and ii) $\mu\left(T_{t_{0}}(U)\right)=\mu\left(T_{t_{0}+t}(U)\right)$ for any subset of $X$ and any $t_{0}$ and $t$. In classical mechanics, condition $\left.i\right)$ is ensured by demanding that space $X$ is the phase space of a finite energy system in a finite box. If $\mu$ is the distribution or density function, $\rho$, in phase space and $T_{t}$ is the evolution operator of the mechanical system (associated with the Hamiltonian or Liouville operator), then condition ii) follows from Liouville's theorem: $d \rho / d t=0$. It thus follows that classical mechanics is inconsistent with the Second Principle of Thermodynamics.

Of course, the recurrence issue was a key concern to Boltzmann, who in the 1870 s realized that deducing irreversibility, an arrow of time, from the mechanics of atoms was impossible without using averaging arguments. It was in the context of his efforts to understand the statistical equilibrium with the Liouville equation that he obtained in 1872 a time-asymmetric evolution equation, now referred to as Boltzmann equation, satisfied by a single-particle distribution function of a molecule in a diluted gas. From this he could construct a mathematical function, the so-called $\mathcal{H}$-function which is a strictly decreasing function of time. Identifying the $\mathcal{H}$-function with entropy with mi- 
nus sign, Boltzmann could claim to have solved the irreversibility problem at molecular level.

However, in order to arrive at his result Boltzmann had to rely on the "molecular chaos hypothesis" (Stosszahlansatz), i.e. on the assumption that molecules about to collide are uncorrelated, but following the collision they are correlated as their trajectories are altered by the collision. Ernest Zermelo (1871 - 1953), young assistant of Planck (1858 - 1947) in Berlin, and Johann Josef Loschmidt (1821 - 1895), friend of Boltzmann, argued that the timeasymmetry obtained by Boltzmann was entirely due to the time-asymmetry of the molecular chaos assumption. Twenty years later, Zermelo attacked Boltzmann once again, now armed with Poincaré's recurrence theorem. Boltzmann attempted to save his case through a cosmological model. He suggested that as a whole the universe had no time direction, but rather individual regions could be time-asymmetric when through a large fluctuation from equilibrium it would yield a region of reduced entropy. These low entropy regions would evolve back to the most likely state of maximum entropy, and the process would repeat itself in agreement with Poincaré's theorem.

Having become clear that a finite system of particles would be recurrent and not irreversible in the long run, Planck considered whether irreversibility could emerge from a field theory such as electromagnetism. The point was to derive irreversibility from the interaction of a continuous field with a discrete set of particles. Starting to tackle the problem in 1897 in a series of papers, the work of Planck culminated with his discovery of the quantum theory of radiation in 1900. From Planck's arguments, Boltzmann remarked that as a field can be regarded as a system with an infinite number of degrees of freedom, and hence expected to be analogous to a mechanical system with an infinite number of molecules, an infinite Poincaré recurrence period and thus agreement with the observed irreversibility and the Second Principle would follow.

However, the persistent objections of influential opponents such as Ernest Mach and Friedrich Ostwald (1853 - 1932), led Boltzmann into depression and a first suicide attempt while at Leipzig before assuming his chair in Vienna in 1902. The intellectual isolation, as he was the sole survivor of the triumvirate of theoreticians along with Clausius and Maxwell, who had developed the kinetic theory of matter, and the continuous deterioration of his health led him to suicide and death at Duino, a seaside holiday resort on the Adriatico coast near Trieste, on the 5 th September 1906. He was 62 years old. Boltzmann death is even more tragic when one realizes that it happened on the very eve of the vindication of his ideas.

However, the irreversibility problem has somehow resisted a straightforward answer. In 1907, the couple Ehrenfest, Paul Ehrenfest (1880 - 1933) and Tatyana Afanasyeva (1876 - 1964), (see e.g. Ref. [33), further developed Boltzmann's idea of averaging over a certain region $\Delta$, of the phase space and showed that the averaged $\mathcal{H}$-function would remain strictly decreasing in the 
thermodynamical limit, after which $\Delta$ could be taken as small as compatible with the uncertainty principle.

In 1928, Pauli (1869 - 1958) considered the problem of transitions in the context of quantum mechanical perturbation theory and showed that consistency with the Second Principle of Thermodynamics would require a "master equation":

$$
\frac{d p_{i}}{d t}=\sum_{j}\left(\omega_{i j} p_{j}-\omega_{j i} p_{i}\right),
$$

where $\omega_{i j}$ is the conditional probability per unit of time of the transition $j \rightarrow i$ and $p_{i}$ is the probability of state $i$. Assuming the $\mathrm{H}$-function to be given by

$$
\mathcal{H}=\sum_{i} p_{i} \ln p_{i},
$$

it follows that $\frac{d \mathcal{H}}{d t} \leq 0$. This approach is quite suggestive as it indicates, as stressed by Boltzmann, that irreversible phenomena should be understood in the context of the theory that best describes microscopic physics.

More recently, Prigogine (1917 - 2003) and collaborators put forward a more radical approach, according to which irreversible behaviour should be already incorporated in the microscopic description (see Ref. 26] for a pedagogical discussion). In mathematical terms the problem amounts to turning time into an operator which does not commute with the Liouville operator, the commutator of the Hamiltonian with the density matrix. In physical terms, this proposal implies that the reversible trajectories cannot be used, leading to an entropy-like quantity which is a strictly increasing function of time.

But, if the problem of explaining the irreversible behaviour of all macroscopic systems from microphysics is already quite difficult, one should realize that there exists in nature quite a variety of phenomena whose behaviour indicate an immutable flow from past to present, from present to future. The term "arrow of time", already used in text, was coined by the British astrophysicist and cosmologist Arthur Eddington (1882 - 1944) 34] to characterize this evolutionary behaviour. Let us briefly describe these phenomena:

1) The already discussed time asymmetry inferred from the growth of entropy in irreversible and dissipative phenomena, as described by the Second Law of Thermodynamics.

2) Nonexistence of advanced electromagnetic radiation, coming from the infinite and converging to a source, even though solutions of this nature are legitimate solutions of the Maxwell's field equations.

3) The collapse of wave function of a quantum system during the measurement process and the irreversible emergence of the classical behaviour, even 
though the fundamental equations of quantum mechanics and statistical quantum mechanics, Schrödinger's and Von Neumann's equations, respectively, are invariant under time inversions for systems described by time-independent Hamiltonians (see e.g. Ref. [35] for a vivid discussion).

4) The exponential degradation in time of systems and the exponential growth of self-organized systems (given a sufficiently large supply of resources). In the development of self-organized systems, a particularly relevant role is played by complexity. The fascinating aspects of phenomena in this context has lead authors to refer to them as "creative evolution", "arrow of life", "physics of becoming" [17, 18, 26, 27. In these discussions, the chaotic behaviour plays an important role given that complex systems are described by non-linear differential equations. This chaotic behaviour gives origin to an extremely rich spectrum of possibilities for describing self-organized systems as well as a paradoxically predictable randomness as chaotic branches are deterministic (see for instance, Refs. [17, 36]).

5) The discovery of the CP-symmetry violation in the $K^{0}-\bar{K}^{0}$ system implies, on account of the CPT-theorem, that the T-symmetry is also violated. This means that on a quite elementary level there exists an intrinsic irreversibility. The violation of the CP-symmetry and also of baryon number in an expanding universe are conditions from which the observed baryon asymmetry of the universe can be established (see e.g. 37] and references therein). An alternative way to achieve the baryon asymmetry of universe is through the violation of the CPT-symmetry (see [38] and references therein).

6) Psychological time is clearly irreversible and historical. The past is recognizable, while the future is open.

7) The so-called gravito-thermal catastrophic behaviour [39] of systems bound gravitationally, implies, given their negative specific heat, that their entropy grows as they contract beyond limit.

On the largest known scales, the expansion of the Universe, which is adiabatic, is a quite unique event, and as such, is conjectured to be the master arrow of time to which all others are subordinated.

\section{Open questions}

Let us briefly discuss in this section a few problems concerning the nature of space-time that remain unsolved. These include the issue of a putative correlation between the above described arrows of time, the problem of nonexistence of an explicit time in the canonical Hamiltonian formulation of quantum gravity, the question of solutions of general relativity and other gravity theories 
that exhibit closed time-like curves and whether the universe evolves after all in a cyclic way.

\subsection{Are the arrows of time correlated?}

The existence of systems, from which a time direction can be inferred, is not on its own very surprising, as it is in the core of all dissipative phenomena. One could argue that this property reflects, for instance, a particular choice of boundary conditions which constrain the state of the universe, rather than any restriction on its dynamics and evolution. However, this point of view cannot account for the rather striking fact that the known arrows of time seem all to point in the same time direction. In the following, we shall briefly overview some of the ideas put forward to relate the arrows of time. Extensive discussions can be found in Refs. [40, 41, 42].

In his book The Direction of Time, the philosopher Hans Reichenbach (1891 - 1953) 43] argued in a rather circular way that the arrow of time in all macroscopic phenomena has its origin in causality, which in turn should be the origin of the growth of entropy. In 1958, the cosmologist Thomas Gold (1920 2004) put forward the remarkable idea that all arrows of time should be subordinated to the expansion of the universe [44. This speculation gave origin to demonstrations, although not quite entirely successful, that the propagation of the electromagnetic radiation was indeed related to the expansion of the universe [45, 46. The problem is that the obtained solutions are somewhat puzzling. Indeed, it is found that: retarded radiation is found to be compatible only with a steady-state universe, while advanced radiation is found to be compatible only with evolutionary universes (expanding or contracting ones). For sure, these solutions indicate that the problem is more complex than admitted.

Inspired by the Thermodynamics of Black Holes, Penrose put forward the suggestion that the gravitational field should have an associated entropy which, in turn, should be related with an invariant combination involving the Weyl tensor [47. Remarkably this suggestion allows for a consistent set up for cosmology of the Generalized Second Principle of Thermodynamics, which arises in black hole physics, and states that the Second Principle should apply to the sum of the entropy of matter with the one of the black hole [48, 49]. The main point of the proposal is that it circumvents the paradox of an universe whose initial state is a singularity or a black hole protected by a horizon, and hence with an initial entropy that exceeds by many decades of magnitude the entropy of the observed universe. Being highly homogeneous and isotropic, the initial state of the universe has necessarily a low entropy 6 as the Weyl tensor vanishes for homogeneous and isotropic geometries. The gravitational entropy will then increase as the Weyl tensor increases as the universe grows lumpier.

\footnotetext{
${ }^{6}$ The low entropy of the highly "excited" and hot initial state was suggested to be analogous of systems with a negative temperature [50].
} 
The growth of the total entropy can presumably account for the asymmetry of psychological time as in this way the branching of states and outcomes will occur into the future.

Let us close this subsection with some remarks on some recent ideas developed in the context of superstring/M-theory. These suggest a multiverse approach of the "landscape" of vacua of the theory (see e.g. [12] and references therein), that is the googleplexus of about $10^{500}$ vacua [51, which are regarded as distinct universes, which asks for a selection criteria for the vacuum of our universe. Anthropic arguments [52] and quantum cosmological considerations 53. have been advanced for this vacuum selection as a meta-theory of initial conditions. These proposals are not consensual, but can be seen as a relevant contribution to a deeper understanding of the problem. Of course, one should keep in mind that non-perturbative aspects of string theory are still poorly known [54]. The multiverse perspective hints at the possibility that different universes may actually interact [55]. It is suggested that this interaction is regulated by a Curvature Principle and shown, in the context of a simplified model of two interacting universes, that the cosmological constant of one of the universes is driven toward a vanishingly small value. The core of this proposal is to set an action principle for the interaction using the curvature invariant $I_{i}=R_{\mu \nu \lambda \sigma}^{i} R_{i}^{\mu \nu \lambda \sigma}$, where $R_{\mu \nu \lambda \sigma}^{i}$ is the Riemann tensor of each universe. The suggested Curvature Principle also hints at a solution for the entropy paradox of the initial state of the universe [55]. For this, one considers the point of view of another universe, from which our universe can be perceived as if all its mass were concentrated in some point and hence $I=48 M^{2} r^{-6}$, where $r$ is the universe horizon's radius and $M$ its mass - using units where $G=\hbar=c=1$. Hence, if the entropy scales with the volume, then $S \sim r^{3} \sim I^{-1 / 2}$; if the entropy scales according to the holographic principle, suitable for AdS spaces [56, 57, then $S \sim r^{2} \sim I^{-1 / 3}$. In either case, given that $I \sim \Lambda^{2}$ for the ground state, one obtains that $S \rightarrow 0$ in the early universe and, $S \rightarrow \infty$ when $\Lambda \rightarrow 0$. The latter corresponds to the universe at late time, which is consistent with the Generalized Second Principle of Thermodynamics.

Of course, a multiverse perspective, if taken to its most extreme versions, can lead to intricate problems concerning the relationship among the cosmic time of each universe and the "meta-time" of the whole network of universes. Only the future will tell us whether developments in this direction will be needed to further understand the physics of our universe.

\subsection{Time in quantum gravity}

Quantum gravity, the theory that presumably describes the behaviour of space-time at distances of the order of the Planck length is still largely unknown. The most developed programme to understand quantum gravity, superstring/M-theory, leads to a quite rich lore of ideas and concepts, but has not provided so far a satisfactory answer concerning for instance, the fundamental problem of smallness of the cosmological constant [58], and exhibits 
the vacuum selection problems discussed above, which seriously threaten the predictability power of the whole approach.

In order to understand the conceptual difficulties of the quantum gravity problem, let us see that from its very beginning, the quantization of gravity poses outstanding challenges to the well known and well tested methods of quantum field theory. Indeed, if one considers the metric, $g_{\mu \nu}(\boldsymbol{r}, t)$, a bosonic spin-two field and attempts its quantization through an equal-time commutation relation for the corresponding operator:

$$
\left[\hat{g}_{\mu \nu}(\boldsymbol{r}, t), \hat{g}_{\mu \nu}\left(\boldsymbol{r}^{\prime}, t\right)\right]=0,
$$

for $\boldsymbol{r}-\boldsymbol{r}^{\prime}$ space-like, then one faces an indefinite problem: i) In fact, in order to establish that $\boldsymbol{r}-\boldsymbol{r}^{\prime}$ is space-like, one must specify the metric; ii) Being an operator relationship, it must hold for any state of the metric; iii) Without specifying the metric, causality is ill-defined.

These difficulties compel one to consider a canonical quantization programme based on Hamiltonian formalism (see e.g. [59] and references therein). In this context, one splits space and time and selects foliations of space-time where the physical degrees of freedom of the metric are the space-like ones, $h_{a b}={ }^{(3)} g_{a b}$. The resulting Hamiltonian is a sum of constraints, one associated with invariance under time reparametrization, the others related with invariance under 3-dimensional diffeomorphisms. If one considers only Lorentzian geometries (a quite restrictive condition!), then only the first constraint is relevant. The solution of the classical constraint is given by:

$$
H_{0}=0,
$$

where

$$
H_{0}=\sqrt{h}\left[h^{-1} \Pi_{a b} \Pi^{a b}-{ }^{(3)} R\right],
$$

$h$ being the determinant of the 3-metric $h_{a b}, \Pi_{a b}$ the respective canonical conjugate momentum and ${ }^{(3)} R$ the 3 -curvature. Quantization follows by turning the momenta into operators for some operator ordering and applying the resulting Hamiltonian operator into a wave function, the wave function of the universe, $\Psi\left[h_{a b}\right]$ :

$$
\hat{H}_{0} \Psi\left[h_{a b}\right]=0 .
$$

This is the well known Wheeler-DeWitt equation.

In this context, the problem of time (see Ref. 60] for a detailed account) consists in not having a Schrödinger-type equation for the evolution of states, but instead, the constrained problem (11), where time is one of the variables within $H_{0}$. Of course, this does not mean that there is no evolution, but rather that there is no straightforward way of extracting a variable from the formalism that resembles the cosmic time one is used to in classical cosmology.

Solutions, although partial, include the semi-classical approach 61, 62, where time is identified with the scale factor or some function of it, once the metric starts behaving like a classical variable and the wave function of the 
universe admits a WKB approximation. In this instance, the Wheeler-DeWitt equation can be written, at least in the minisuperspace approximation, as the Hamilton-Jacobi equation for the action of the WKB approximation. Physically it implies that time is meaningful only after the metric becomes classical.

Another interesting idea is the so-called "Heraclitean time proposal" 63 , 64. This is based on a suggestion by Einstein [65] according to which the determinant of the metric might not be a dynamical quantity. In this theory, usually referred to as unimodular gravity, the cosmological constant arises as an integration constant and an "Heraclitean" time can be introduced as the classical Hamiltonian constraint assumes the form [63]:

$$
H=\Lambda h^{1 / 2},
$$

and thus, for a given space-like hypersurface $\Sigma$, one can write

$$
i \frac{\partial \Psi}{\partial t}=\int_{\Sigma} d^{3} x h^{-1 / 2} \hat{H}_{0} \Psi=\hat{H} \Psi,
$$

which has a Schrödinger-like form.

For sure, the problem of time in quantum gravity still remains an open question and the above approaches were presented only to exemplify some possible directions for future research.

\subsection{Closed time-like curves and time travel}

As already mentioned, closed time-like curves arise as solutions of Einstein's field equations. These solutions include traversable wormholes [22, 66, 67, 68, warp drives [69, 70] and the Krasnikov tube [71]. One can argue that they are unphysical as they violate the energy conditions 72. These solutions correspond to putative forms of time travel and most often bring a host of paradoxes of the ancestor's murder type. However, given that the murder of an ancestor by a time traveler should be logically inconsistent, one could ask whether there should exist global self-consistent conditions to exclude closed time-like curves. These conditions are referred to as consistency constraints. The most discussed of these consistency constraints are the Principle of SelfConsistency [73] and the Chronology Protection Conjecture [74].

The Principle of Self-Consistency states that events along a closed timelike curve are self-consistent, that is they influence each other, but in a selfconsistent fashion. Of course, along a closed time-like curve the notion of past or future is ambiguous and the causal structure of usual space-times is meaningless. The self-consistent condition establishes that events in the future can influence events in the past, but cannot alter them. Hawking's Chronology Protection Conjecture is based on the experimental evidence that "we have not been invaded by hordes of tourists from the future" 74 from which it is then conjectured that the renormalized stress-energy tensor quantum expectation values diverge as they approach closed time-like curves. This divergent 
behaviour destroys the wormhole's structure before the Planck scale is attained. So far, no proof of this conjecture is available.

Thus, one sees that the reality of closed time-like curves may be contested on physical as well as on logical grounds. Nevertheless, these solutions are vivid examples of the wealth of structurally distinct solutions of general relativity and show how some classes of solutions may require a specific set of criteria to establish their physical reality.

\subsection{A cyclic time ?}

The general theory of relativity allows a for a global dynamical description of the physical space-time and for a relation with the history and evolution of the universe. The mathematical description of space-time admits a wide range of scenarios, which includes solutions with cyclic nature. Already in 1922 Alexander Friedmann (1888 - 1925), the first to study evolving cosmological solutions within general relativity, realized that cyclic scenarios existed among his solutions. These involved an expanding universe followed by a recollapse so that the universe's radius would eventually vanish from which a new expansion would ensue. Of course, strictly speaking these cycles are not mathematically admissible as they are disjoint by a singularity. In 1931, Richard Tolman (1881 - 1948) [75] showed that such discontinuity was unavoidable at the beginning and at the end of any isotropic and homogeneous closed geometries for a physically realistic energy-momentum tensor. Subsequently, he argued that the problem was actually due to the highly symmetric nature of the studied solutions and that in a physically realistic universe the discontinuity could very well disappear 76 .

A cyclic or "phoenix" universe was regarded with sympathy by Einstein and George Gamow (1904 - 1968), who even coined the term "big squeeze" to denote the final state of collapse - nowadays the term "big crunch" is more used. Of course the issue of space-time singularities was not fully appreciated then; however, in the 1960s it was understood, through the Hawking-Penrose singularity theorems, that the conditions and the generality of the difficulty could not be overlooked and cosmologists had to accept the reality of the space-time singularities. Some relativists argued however, that quantum effects could play a role in the process of "bouncing" at very high densities completing in this way the cycle of a closed universe. John Wheeler, for instance, advocated that in the "bounce" physical constants would be recycled 77.

More recently, developments in string theory and the related dynamics of branes do open the possibility of reviving the idea of a cyclic universe. In the so-called "ekpirotic" model [78, one assumes, as a starting point, the existence of two 3-dimensional parallel branes embedded in a higher dimensional space. Our universe corresponds to one of these branes. Quantum fluctuations in the other brane would lead to the creation of a third brane, which would be attracted to ours. The ensuing impact of the third brane into ours would trigger 
a release of energy, ekpirosis in Greek, giving origin to a proto-universe, whose subsequent expansion would have properties similar to the ones of a universe just emerging from the inflationary process. Thus, this collision process is quite similar to the Big-Bang itself. Of course, whether a universe emerging from the ekpirotic process fully resembles our universe, or whether it advantageously replaces the inflationary dynamics, whose most generic features are consistent with the latest observations of the cosmic microwave background radiation [79, is still a quite open question. It is interesting that these two competing models have a distinct behaviour in what concerns the production of primordial gravitational waves. The ekpirotic process tends not to produce too much gravitational waves, while some models of inflation do produce a considerably greater amount of gravitational radiation. The possibility of verifying the prediction of these models through the observation of gravitational waves is of course quite exciting.

The cyclic nature of the "ekpirotic" model arises from the fact that after several decades of thousands of millions of years after the brane collision, our universe will expand to the point where stars and galaxies will be all gone and there will be no radiation left. This void and cold brane will be very similar to the one before the Big-Bang. Conditions will then be favourable for the creation of a third brane from the other original brane and the whole process then repeats itself 7 .

\section{Conclusions and Outlook}

The unification of space and time proposed by Minkowski a century ago allowed for an elegant formulation of the special theory of relativity, and was the culmination of more than two thousand years of philosophical and physical research on the nature of space and time. The space-time continuum is a basic foundational concept in physics, from elementary particle physics to cosmology. The vector space structure of the space-time continuum made the transition to the general theory of relativity smooth and quite logical once it was understood that, at cosmological scales, the space-time continuum was not an a priori concept, independent of the physical conditions. Furthermore, when analyzing the inner makings of matter, Minkowski's space-time formulation, together with quantum mechanics, made possible, through renormalizable quantum field theory, to stretch our knowledge down to scales of about $10^{-18} \mathrm{~m}$. The research on the matching of general relativity with the quantum nature of matter is still in its infancy; however, we already understand that reconciling these two pillars of the XX's century physics will require a whole new set of ideas, as it may happen, that we may have to give up concepts that were supposed to be the starting point of all the modeling of the universe,

\footnotetext{
7 The similarity with the Indian mythology is compelling. Each cycle is analogous to the "day of Brahma". The whole process resembles the "life of Brahma".
} 
such as that space-time is a continuum and that the fundamental building blocks of matter are not point-like particles.

These assumptions lead to quite new realms for research and experimentation. They also pose us new technical and conceptual problems. These imply that the very principles upon which our theories of space-time were built so far, such as Lorentz invariance, CPT-symmetry, the commutative nature of the fundamental dynamical variables and so on, will have to be continuously scrutinised. Their breakdown may provide important insights into the nature of the new theories of space-time, matter and the universe. Of course, these new theories will have to match smoothly our current physical theories and explain the conditions for the emergence of the Minkowski space-time continuum as well as to set the boundaries of validity of general relativity and the emergence of the classical features of gravity. The new theories will, like in the case of general relativity, pose questions of ontological nature and should set criteria for selecting, among the mathematically consistent solutions, the physical ones which have predictive power to explain our world. The most recent developments in the context of superstring/M-theory, the most studied quantum gravity programme, suggest that a multitude of universes is needed to explain the physics of our universe. This is a somewhat disappointing outcome for a theory that naturally unifies quantum mechanics and general relativity. However, this may only reflect the provisional state of our knowledge.

On the other hand, as we have discussed, the quest for the understanding of the ultimate nature of space-time, and the rather special role played by time in macrophysics and its various arrows is still largely unknown. If all arrows of time can be related with the expansion of the universe, or to some new curvature principle that properly accounts for the entropy of the gravitational field, a remarkable new unification could be achieved.

In any case, the quest for the ultimate theories about the nature of space and time have mesmerized human thought for more than two thousand years. Till recently, the most insightful ideas sprang from philosophical speculation, however since the pioneering work of Einstein on relativity and the space-time unification proposed by Minkowski a century ago, physicists have taken the lead in this search. A hundred years after the proposal of Minkowski, mankind is about to embark on new expeditions to conquer new continents of knowledge through new scientific challenges which include the Large Hadron Collider to search for the nature of mass and new symmetries, and new space missions to study the polarization of the cosmic microwave background radiation and to directly detect gravitational waves. It is the hope of the whole scientific community that the outcome of these experiments will bring precious hints for the understanding of our universe. 


\section{References}

1. H. Minkowski Raum und Zeit, 1908. Also in A.H. Lorentz, A. Einstein e H. Minkowski, "O Princípio da Relatividade" (Fundação Calouste Gulbenkian, Lisboa 1978).

2. V. Petkov, "Relativity, Dimensionality, and the Existence", in Relativity and the Dimensionality of the World, Ed. V. Petkov, Springer Fundamental Theories of Physics 153 (Springer, AA Dordrecht The Netherlands 2007).

3. H. Müller et al. Phys. Rev. Lett. 91, 020401 (2003); P. Wolf et al., Phys. Rev. Lett. 90, 060402 (2003).

4. O. Bertolami and C. Carvalho, Phys. Rev. D. 61, 103002, (2000).

5. O. Bertolami and D.F. Mota, Phys. Lett. B. 455, 96, (1999).

6. G.F.R. Ellis and J.P. Uzan, "c is the speed of light, isn't it?", gr-qc/0305099.

7. M. Consoli and E. Costanzo, "Is the physical vacuum a preferred frame?", arXiv:0709.4101[hep-ph].

8. D. Colladay and V.A. Kostelecký, Phys. Rev. D. 55, 6760 (1997); D. 58, 116002 (1998).

9. CPT and Lorentz Symmetry III, Alan Kostelecký, ed. (World Scientific, Singapore, 2005); O. Bertolami, Gen. Rel. Gravitation 34, 707 (2002); O. Bertolami, Lect. Notes Phys. 633, 96 (2003) 96, hep-ph/0301191; D. Mattingly, Liv. Rev. Rel. 8,5 (2005), gr-qc/0502097; R. Lehnert, "CPT- and Lorentz-symmetry breaking: a review", hep-ph/0611177.

10. Abraham Pais, "Subtil é o Senhor, Vida e Pensamento de Albert Einstein", (Gradiva, Lisboa 2004).

11. S.W. Hawking and G.F.R. Ellis, "Large Scale Structure of Space-Time" (Cambridge University Press, 1973).

12. O. Bertolami, "The adventures of Spacetime", in Relativity and the Dimensionality of the World, Ed. V. Petkov, Springer Fundamental Theories of Physics 153 (Springer, AA Dordrecht The Netherlands 2007).

13. C. Will, "The Confrontation between General Relativity and Experiment", grqc/0510072.

14. O. Bertolami, J. Páramos and S. Turyshev, "General Theory of Relativity: Will it survive the next decade?", gr-qc/0602016.

15. B. Russell, "History of Western Philosophy" (Counterpoint, London 1946).

16. N. Abbagnano, "História da Filosofia" (Editorial Presença, Lisboa 1984).

17. P. Coveney and R. Highfield, "The Arrow of Time" (Fawcett Columbine, New York 1990).

18. "Des hommes de science aux prises avec le temps", Group de Matheron (Presse Polytechniques e Universitaires Romandes, Laussane 1992).

19. G.F.R. Ellis, "Physics in a Real Universe: Time and Space-Time", in Relativity and the Dimensionality of the World, Ed. V. Petkov, Springer Fundamental Theories of Physics 153 (Springer, AA Dordrecht The Netherlands 2007).

20. O. Bertolami, "The concept of time in physics", in Proceedings of the 7th International Conference of Physics Students, Lisbon 1992.

21. O. Bertolami, "O Livro das Escolhas Cósmicas" (Gradiva, Lisboa 2006).

22. F.S.N. Lobo, "Nature of time and causality in Physics", arXiv:0710.0428 [gr-qc].

23. P. Candelas, G. Horowitz, A. Strominger and E. Witten, Nucl. Phys. B 258, $46(1985)$

24. E. Witten, Nucl. Phys. B 443, 85 (1995). 
25. A. Ashtekar, C. Rovelli and L. Smolin, Phys. Rev. Lett. 69237 (1992).

26. I. Prigogine, "From Being to Becoming" (W.H. Freeman \& Co. 1980).

27. I. Progine and I Stengers, "La Nouvelle Alliance" (Gallimard, Paris 1979).

28. E.T. Bell, "Men of Mathematics" (Simon and Schuster, New York 1965).

29. Orphica, Fragments, Greek Hymns C3rd B.C. - C2nd A.D.

30. F.J. Tipler, Essays in General Relativity, Festschrift for A. Taub, Ed. F.J. Tipler (Academic Press, 1980).

31. J.L. Borges, "El Aleph" (Emecé Editores, Buenos Aires 1957).

32. W. Shakespeare, "The Illustrate Stratford Shakespeare" (Chancellor Press, London 1982).

33. K. Huang, "Statistical Mechanics" (John Wiley, New York 1966).

34. A. Eddington, "The Nature of the Physical World" (Cambridge University Press, 1928).

35. R. Penrose, "The Emperor's New Mind" (Vintage, London 1990).

36. J. Gleick, "Chaos" (Cardinal, London 1988).

37. W. Buchmüller, "Baryogenesis - 40 Years later", arXiv:0710.5857[hep-ph]

38. O. Bertolami, D. Colladay, V.A. Kostelecký and R. Potting, Phys. Lett. B. 395, 178 (1997).

39. D. Lynden-Bell, Mont. Not. Roy. Astr. Soc. 123, 447 (1962).

40. P.C.W. Davies, "The Physics of the Time Asymmetry" (California University Press, Berkeley 1974).

41. H.D. Zeh, "The Physical Foundation of the Direction of Time", Heidelberg University Preprint 1988.

42. M. Gell.Mann and J. B. Hartle, "Time Symmetry and Asymmetry in Quantum Mechanics and Quantum Cosmology", University of California Santa Barbara Preprint 1991.

43. H. Reichenbach, "The Direction of Time", (California University Press, Berkeley 1956).

44. T. Gold, "La Structure et L'Evolution de L'Universe", 11th International Solvay Congress (Edition Stoops, Brussels 1958).

45. J.E. Hogarth, Proc. R. Soc. London A 267, 365 (1962).

46. F. Hoyle and J.V. Narlikar, Proc. R. Soc. London A 277, 1 (1964).

47. R. Penrose, in "General Relativity: An Einstein Centenary Survey", Eds. S. Hawking and W. Israel (Cambridge University Press, 1979).

48. R. Penrose, Il Nuovo Cimento, Num. Spec. I 1, 252 (1969).

49. J.D. Bekenstein, Phys. Rev. D. 7, 2333 (1973); D. 9, 3292 (1974); S.W. Hawking, Comm. Math. Phys. 43, 199 (1975).

50. O. Bertolami, "Negative Temperatures and the arrow of time" (1985); unpublished.

51. R. Bousso and J. Polchinski, JHEP 0006,006 (2000).

52. L. Susskind, "The Anthropic Landscape of String Theory", hep-th/0603249; L. Susskind, "The cosmic landscape: String theory and the illusion of intelligent design" (Little, Brown, New York 2005).

53. R. Holman and L. Mersini-Houghton, "Why the Universe Started from a Low Entropy State", hep-th/0511102;

R. Holman and L. Mersini-Houghton, "Why did the Universe Start from a Low Entropy State ?", hep-th/0512070.

54. J. Polchinski, "The Cosmological Constant and the String Landscape", hepth/0603249. 
55. O. Bertolami, "A Curvature Principle for the interaction between universes", arXiv:0705.2325[gr-qc]; to appear in General Relativity and Gravitation.

56. W. Fischler and L. Susskind, "Holography and Cosmology", hep-th/9806039.

57. R. Bousso, JHEP 9906, 028 (1999).

58. E. Witten, "The Cosmological Constant From The Viewpoint Of String Theory", hep-ph/0002297.

59. O. Bertolami and J. M. Mourão, Class. Quant. Grav. 8, 1271 (1991).

60. C. Isham, "Canonical quantum gravity and the problem of time", grqc/9210011.

61. B.S.DeWitt, Phys. Rev. 160, 1113 (1967).

62. A. Vilenkin, Phys. Rev. D. 39, 1116 (1989).

63. W. Unruh, Phys. Rev. D 40, 1048 (1989);

W. Unruh and B.M. Wald, Phys. Rev. D 40, 2598 (1989).

64. O. Bertolami, Int. J. Mod. Phys. D 4, 97 (1995).

65. A. Einstein, Sitz. Berl. Preuss. Akad. Wiss. (1919).

66. M. Visser, "Lorentzian Wormholes: From Einstein to Hawking", (American Institute of Physics, New York 1995).

67. M. Morris and K.S. Thorne, Am. Journ. Phys., 56, 395 (1998).

68. F.S.N. Lobo, Phys. Rev. D 71, 084011 (2005); D 71, 124022 (2005).

69. M. Alcubierre, Class. Quant. Grav. 11, L73 (1994).

70. F.S.N. Lobo and M. Visser, Class. Quant. Grav. 21, 5871 (2004).

71. S.V. Krasnikov, Phys. Rev. D 57, 4760 (1998).

72. M. Morris, K.S. Thorne and U. Yurtsever, Phys. Rev. Lett. 61, 1446 (1988);

S. Deser, R. Jackiw and G. t'Hooft, Phys. Rev. Lett. 68, 267 (1992);

S. Deser, Class. Quant. Grav. 10, S67 (1993).

73. J. Earman, "Bangs, Crunches, Whimpers and Shrieks: Singularities and Acausalites in Relativistic Spacetimes" (Oxford University Press, 1995).

74. S.W. Hawking, Phys. Rev. D 56, 4745 (1992).

75. R. Tolman, "Relativity, Thermodynamics and Cosmology" (Oxford University Press, 1934).

76. R. Tolman, Phys. Rev. 38, 1758 (1931).

77. C.W. Misner, K.S. Thorne and J.A. Wheeler, "Gravitation" (Freeman, San Francisco 1973).

78. N. Turok and P. Steinhard, "Beyond Inflation: A Cyclic Universe Scenario", hep-th/0403020.

79. D.N. Spergel et al. [WMAP Collaboration], Astrophys. J. Suppl. 170, 377 (2007). 